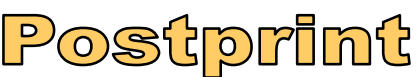

Version définitive du manuscrit publié dans / Final version of the manuscript published in : Ecological Complexity, 2009, DOI: 10.1016/j.ecocom.2009.03.012

1

2

$3 \quad$ André Kretzschmar ${ }^{1, *}$, Samuel Soubeyrand ${ }^{1}$ and Nicolas Desassis ${ }^{2}$

${ }_{4}{ }^{1}$ INRA, Unité Biostatistique et Processus Spatiaux, Agroparc, F-84914 Avignon, France

$5{ }^{2}$ MERE project, INRIA/INRA, UMR INRA/SupAgro Analyse des Systèmes et Biométrie, 2 6 place Viala, F-34060 Montpellier, France

7 * Corresponding author: andre.kretzschmar@avignon.inra.fr - FAX : +33(0)432722182

\section{Aggregation patterns in hierarchy/proximity spaces}

\section{Abstract}

The classical approaches for modelling spatial structures address aggregation through a set of distributional hypotheses. They require a large number of data and the definition of a spatial distance for the characterization of spatial correlations. When only scarce abundance data and no Euclidean distance are available, we propose a method to explore the structuring patterns by the association of a hierarchical/proximity description of the space under study (units formed by subunits) and a multiple permutation test battery capturing the similarity between units (permutation being applied at subunits or units level). No Euclidean distances being available among the elements of a hierarchical level, proximity will be used instead of distance and will be defined by the structural relationships of units or subunits. The multiple test aims to assess different types of structuring, determined either by occupancy or by abundance or by both, at unit or subunit level.

We address the relevancy of this approach with a simulation study where samples, formed by ordered subunits and units, are designed to exhibit the different types of structuring. The method is also illustrated with a case study (distribution of aphids on 


\section{Keywords}

Citrus branches). It results from this application a decomposition of the complexity of pattern structuring and the evidence, for a single species in its natural environment, to develop diverse types of pattern structuring. Additionally to their direct implications, the results of the test battery may provide valuable preliminary hypotheses and could serve as a constraining context for the next step of modelling.

density singularity ; permutation tests ; simulation studies ; aggregate scale ; aggregation patterns ; complexity decomposition.

\section{Introduction}

\subsection{Capturing aggregation characteristics}

Biological populations present spatial patterns which could be regarded as signatures of population dynamics responding to environmental heterogeneity (Levin, 1992) and to limitation in spatial connectivity and dispersal. According to this general statement, characterising the patterns can help to identify settlement strategies of populations in their environments (Grimm et al., 2005; Wiegand et al., 2003).

Presently, under different names ("clustering", "structuring", "spatial structures or patterns", "aggregation", etc...), spatial structurings are described using different approaches with specific adaptations to each case study (see, for instance : Tilman et al., 1996; Perry et al., 2002; Johst et al., 2002; Keitt et al., 2002; Gilbert et al., 2003; Inouye, 2005, Conlisk et al., 2007).

Such a variety of approaches points out the difficulty of characterising how living beings exhibit spatial structuring and leads to take into account that structuring of populations should not be studied out of the context of specific populations strategies and out of environmental spatial organisation. But efficient models of these strategies in order to provide comprehensive representation of structuring patterns are "far to be 
1 reached" (Baddeley, 2008). As a mid step study, it is nevertheless possible to focus on 2 the complexity of patterns and to expect that the decomposition of this complexity leads to elaborate realistic and accurate models of population dynamics.

The future modeling we have in mind will reflect the population strategy of colo5 nization; in this case, aggregation would be regarded in a spatio-temporal perspective.

6 The difficulty to directly cope with such a modeling leads us to apply a pattern-oriented 7 modeling approach (Grimm et al., 2005; Wiegand et al., 2003). The question is then to s define the pattern characteristics (also called summary statistics) which will allow the analyst to point out the main components of colonization strategies. In other words, we aim to capture the different scales and hierarchical levels at which structuring can be depicted (Bar Hen and Picard, 2007).

\subsection{Aggregation in hierarchy/proximity spaces}

Classical methods used for studying aggregation are standardly applied for physical spaces with Euclidean distances; e.g. variogram, auto-covariance, Moran's index plotted against distance (Chilès and Delfiner 1999; Cressie, 1991), deviance from complete spatial randomness (CSR), $K$-function (Diggle, 2003, Illian et al., 2008).

However, situations arise where a distance cannot be defined in a univocal manner. Consider a tree attacked by aphids for example, how to define a distance between aphids settled on leaves carried by shoots nested in branches? Do we use the Euclidean distance in the 3D space? Do we use a distance measured (in meters or number of leaves) along shoots and branches? In such a situation, instead of defining a distance stricto senso, we propose to characterize aggregation by resorting to the more robust notions of hierarchy and proximity.

The hierarchy attached to a given portion of space (a piece of branch for example) is defined when, in this space, it is possible to identify several units, each of one being divided into sub-units. For example : leaves are nested in shoots, shoots are nested in branches. When no Euclidean distances are available among the elements of a hierarchical level, proximity will be used instead of distance and will be defined by the structural 
relationships of units or sub-units ; for example, leaves are ordered in a shoot, shoots are ordered in a branch; see Fig. 1.

(Figure 1 about here)

This hierarchy/proximity scheme is shared by many data sets in population ecology (Virtanen et al., 1996 , Henderson et al., 2002; Oddou et al., 2004; Laine and Hansky, 2006; Soubeyrand et al., 2007a, 2007b, 2008b). Note that the proximity can be a temporal proximity. For instance, consider a set of habitats for a given species and suppose that the abundance is measured annually at the end of the growing season in each habitat; in this case, the annual observations are the units, the units are ordered in time, and the subunits are the habitats (see Laine and Hanski, 2006, for such a data set).

To sum up the problem, we aim to characterize aggregation in hierarchical spaces (i) which contain subunits nested in units, (ii) where a relation of proximity between units exists (not necessarily between subunits) and (iii) where population abundance is measured at the subunit level. It has to be noted that in a space made of many levels of hierarchy, aggregation can be studied at different scales by applying, to different couples of levels, the procedure here proposed.

In the above defined hierarchy/proximity spaces, aggregation is considered in two ways. First, a spatial pattern is regarded with a binary filter: each subunit is weighed either by the value 0 or the value 1 describing a status (absence or presence of a species, abundance less or greater than a given threshold). Thus, aggregation is a nonrandomness in occupancy. Second, abundance is studied as a quantitative variable and aggregation is due to abundance singularities among the subunits which are occupied. Unoccupied subunits are excluded at this step because if not, a non-randomness in abundance can be only due to a non-randomness in occupancy. Doing so, we aim to filter out the patterns related with occupancy.

Both forms of non-randomness and their combinations are illustrated by Fig. 2 . Third, aggregation in occupancy and/or in abundance can take place at various scales in the sampled space (within a unit, over several units - how many?). In addition, aggregation can be modulated because of the irregularity or fragmentation of the space. 
1 Here, we are concerned by the variation in the number of subunits per unit.

(Figure 2 about here)

\subsection{Analysing aggregation with a multiple test procedure}

4 The detection and characterisation of non-randomness and the determination of the scale

5 of objects on which non-randomness is based require the choice of appropriate statistical

6 tools. To meet this aim, we characterise a spatial pattern observed on a hierarchical 7 space by its multivariate response to a set of permutation tests (Manly, 1997) made at 8 various scales. The set of tests is diverse enough to tackle non-randomness in occupancy, 9 non-randomness in abundance, and variation in scale of aggregation. Using permutations allows us to account for the irregularity (or fragmentation) of the space. The method allows us to capture the possible complexity of aggregation.

In what follows, we present the multiple test procedure and apply it on simulated data and real data. Carrying out the procedure for simulated data shows what multivariate responses to the test battery are obtained with diverse forms of aggregation. The real data describes spatial patterns of aphids in branches sampled on citrus trees. Each branch contains 10 ordered shoots and each shoot contains a varying number of leaves on which aphids were counted. The application of the method to this data set allows us to decompose the complexity of aphid aggregation. For the sake of clarity, we built the method and applied it when the proximity between units is just a matter of order (shoots are distributed (ordered) on a 1D line, i.e. the branch). However, at the end of this communication, we consider the application of the method in more complex configurations. In addition, we discuss the decomposition of complex forms of aggregation. 


\section{Methods : Multiple test procedure}

\section{$2 \quad 2.1$ From density singularities to randomness hypotheses}

3 Although the definition of an aggregate is as diverse as the object to which it is applied, 4 it is commonly accepted that aggregation is associated to singularities (unexpected high 5 values) among a density field. A density singularity reflects a departure from a null hypothesis which governs, in our context, (i) what are the abundances of individuals in the subunits and (ii) how these abundances are structured in the space. Under the complete spatial randomness (CSR) hypothesis, a classical null hypothesis in spatial statistics (Diggle, 2003, Illian et al., 2008), individuals are settled independently and uniformly on leaves; consequently, the numbers of individuals in subunits are drawn from independent Poisson distributions with a common expected value.

This hypothesis is particularly strong and it can be relevant to consider lighter null hypotheses under which, for instance, the abundances are not Poissonian and/or the subunits are not equally suitable to settlement of individuals. Using other null hypotheses prevent us to detect aggregates which are not interesting in our context (e.g. aggregates at the subunit scale). The reasons why an aggregate should be detected as such depend on the phenomena investigated and questions of interest. Three null hypotheses of Partial Spatial Randomness were considered. They are not defined through a settlement process as the CSR hypothesis is, but through permutations of space pieces with their numbers of individuals. The permutations (Manly, 1997) allow us to preserve the marginal distribution of individual abundances without making any distribution assumption such as the Poisson one made under the CSR hypothesis. Specific choices for the nature and the size of the permuted space allow us to focus on different types of aggregates (non-randomness in occupancy, in abundance, and at given scales). Notice again that we consider spaces made of units, themselves made of subunits. The three null hypotheses that we propose are defined as follows.

- $H_{01}$ : the observed pattern of occupancy (individual presence/absence) conforms with the distribution of patterns obtained when all the subunits are permuted (see 
Fig. 3, top). Under this hypothesis, the probability of presence of individuals in a subunit is independent of the occupancy pattern around it. In other words, there is no spatial structure in the subunit occupancy pattern.

- $H_{02}$ : the observed pattern of abundances conforms with the distribution of patterns obtained when only the occupied subunits are permuted (see Fig. 3, centre). Under this hypothesis, abundances are not spatially structured on the subset of occupied subunits irrespectively to the spatial pattern corresponding to $H_{01}$. This hypothesis will be tested to investigate the abundance pattern conditional on the occupancy pattern.

- $H_{03}$ : the observed pattern of abundances conforms with the distribution of patterns obtained when the units are permuted (see Fig. 3, bottom). Under this assumption, abundances are not structured at the scale of several units irrespectively to the spatial pattern corresponding to $H_{01}$ and $H_{02}$ for a single unit.

Departures from $H_{01}$ or $H_{02}$ can reflect similarity between close subunits, and a departure from $H_{03}$ can reflect a similarity between close units.

(Figure 3 about here)

\subsection{Statistics and tests}

In the previous paragraph, we defined null hypotheses with the aim to detect the existence of specific types of aggregates. We now provide statistics which were used to test if the observed patterns conform or not with the patterns obtained by permutations under the null hypotheses.

We used statistics measuring the similarity between neighbour subunits or units in the zone where an aggregate is searched for. Additionally, in order to capture the size which characterizes a given aggregate, the test was sequentially applied to groups of units, called uplets, corresponding to increasing numbers $n$ of close units.

For the sake of simplicity, we assume in the following that the units are distributed in a space along one dimension. Thus, the proximity relationship between units simplifies 
1 into an order relationship. The case of several dimensions is discussed at the end of this 2 article. When units are ordered, a $n$-uplet is a group of $n$ successive units. In a space of $q$ units, the number of 2 -uplets is $q-1$, the number of 3 -uplets is $q-2$ and so on.

The test statistics is defined here. Consider a pattern of individuals observed on a 5 space made of $q$ ordered units in which are nested various numbers of subunits. Let $n$ be 6 a fixed uplet size. Let $A_{n k}$ denote the set of abundances (counts) or occupancies (binary 7 indices $0 / 1$ for absence/presence) measured in subunits belonging to the $k$-th uplet of 8 size $n\left(1 \leq k \leq K_{n}\right.$ where $K_{n}$ is the number of $n$-uplets). Let $\mu_{n k}$ and $\sigma_{n k}^{2}$ denote, 9 respectively, the sample mean and the sample variance based on $A_{n k}$. The test statistic $S_{n}$ is the mean-variance pair corresponding to the maximum mean over the $K_{n} n$-uplets

$$
S_{n}=\left(\mu_{n k^{\star}}, \sigma_{n k^{\star}}^{2}\right) \quad \text { with } \quad k^{\star}=\underset{1 \leq k \leq K_{n}}{\operatorname{argmax}} \mu_{n k} .
$$

Figure 4 illustrates the computation of $S_{n}$ for an hypothetical space with four units and observed at the scale of abundances in subunits. A similar computation can be performed when the space is observed at the scale of occupancies in subunits or at the scale of abundances in units.

(Figure 4 about here)

With the statistic $S_{n}$, we focused on the $n$-uplet with the highest abundance of individuals (using the maximum mean) and we look at its homogeneity (using the variance); an homogeneous uplet with a high abundance may reflect an aggregate. So, $S_{n}$ characterises what could be the aggregate at the scale of n-units. The following procedure based on permutations allows us to determine the significance of this potential aggregate (if there is an aggregate at any uplet size $n$, then there is aggregation in the pattern under study).

For any uplet size, each of the three null hypotheses can be tested as follows. The mean-variance statistic proposed above is computed for the observed pattern and for 5000 permuted patterns obtained under the null hypothesis. This yields a cloud of points (one observed point plus 5000 so-called permuted points) spread in the mean-variance plane (see Fig. 5). The presence of an aggregate in the observed pattern should result in a 
1 point significantly aside from the permuted points. A p-value is then computed in order 2 to measure the departure of the observed point from the simulated distribution. For each point $j$ (the observed point $j=0$ and the 5000 permuted points $j=1, \ldots, 5000$ ), the proportion of permuted points for which $N_{j}$ is less or equal than $N_{0}$ :

$$
\frac{1}{5000} \sum_{j=1}^{5000} \delta\left(N_{j} \leq N_{0}\right),
$$

where $\delta\left(N_{j} \leq N_{0}\right)=1$ if $N_{j} \leq N_{0}$ and zero otherwise. The test is one-tailed because we aim to only detect the points which are aside from the cloud.

(Figure 5 about here)

Assume that $N$ different sizes of uplets are taken into consideration, as there are three null hypotheses, a set of $3 \times N$ p-values describes a studied pattern. Thus, a pattern has a signature in a $3 N$-dimensional space allowing (i) the identification of density singularities that we associated with aggregates, and (ii) the differentiation between various types and sizes of density singularities.

\section{Results}

\subsection{Simulation methods}

In order to assess the pertinence of the tests proposed here, a simulation study has been performed. Four types of simulated samples have been built expected to reject specific null hypotheses. They were achieved (see Appendix) by combining three layers: (i) a hierarchical space $\mathbb{E}$, (ii) a structure in occupancy $O: \mathbb{E} \rightarrow\{0,1\}$ defined over $\mathbb{E}$ and 
1 with value zero or one, and (iii) a structure in abundance $A: \mathbb{E} \rightarrow \mathbb{N}$ defined over $\mathbb{E}$ and 2 with integer values.

- An $O A$-sample, structured in occupancy and abundance, was obtained by multiplying the two structures $O$ and $A$ (i.e. the abundance at any subunit $e$ in $\mathbb{E}$ is $O(e) * A(e))$.

- An $O$-sample, structured in occupancy only, was obtained by multiplying $O$ and a random permutation of $A$.

- An $A$-sample, structured in abundance only, was obtained by multiplying $A$ and a random permutation of $O$.

- A $\emptyset$-sample, with no structure, was obtained by multiplying a random permutation of $O$ and a random permutation of $A$.

For each of this type, 100 samples have been simulated and then, each of them was submitted to the three test battery. Fig. 6 (four top panels) illustrates the types of patterns which are obtained.

(Figure 6 about here)

\subsection{Multiple test results}

For each type of simulated samples, the counts of samples (among 100) with p-values less than 0.05 are provided for the three hypotheses and the nine uplet-sizes. If the null hypothesis is satisfied, five tests among 100 are expected to be rejected. A star marks the counts which are significantly higher than this value, with the risk of 0.05 (the binomial distribution $B(100,0.05)$ was used to assess the significance).

(Table 1 about here)

As general results, it is pointed out :

- the multiple test provided the right description of the type of structuring which have been simulated (see Tab. 1): in general, $H_{01}$ and $H_{02}$ are rejected simultane- 


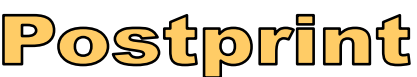

ously for $O A$-samples, $H_{01}$ is rejected for $O$-samples, $H_{02}$ is rejected for $A$-samples and neither $H_{01}$ nor $H_{02}$ are rejected for $\emptyset$-samples.

- additionally, it is to be noticed that, in all types, $H_{\text {оз }}$ is never rejected. We face here a lack of power when testing $H_{03}$. By permuting only ten units, we were not able to detect medium scale aggregates as shown in Fig. 6 (top right) where two dense aggregates have been nevertheless simulated. To better understand this particular problem, samples with $L A R G E$ aggregates (a single aggregate covering about five units) have been simulated (see Fig. 6, bottom). As shown in Table 1, $H_{03}$ is rejected for 4 - or 5-uplets. However the lack of power is still noticeable $\left(H_{03}\right.$ is rejected only 15 and 23 times over 100 for, respectively, 4- and 5-uplets).

- in Tab. 1 , the frequencies of test rejections decrease with uplet size for $H_{01}(O A$ and $O$-samples $)$ and $H_{02}(O A$ - and $A$-samples). This decreasing trend reflects that small and medium scale aggregates have been simulated in these samples. When large aggregate samples are simulated, another trend is seen: the frequencies of rejection of $H_{01}$ and $H_{03}$ first increase and then decrease; the frequency of rejection of $H_{02}$ is first constant and then decreases.

- Tab. 1 does not provide the association between hypotheses which would allow us to depict, for example, that aggregates in occupancy are always associated with aggregates in abundance or, on the contrary, that these two types of aggregates are never on the same space. To study such associations, we can consider the trivariate responses to the test of $\left(H_{01}, H_{02}, H_{03}\right)$. Instead of looking to these responses for each n-uplet, we summarized information over the n-uplets: for instance, the response $(1,0,0)$ is obtained for a sample for which $H_{01}$ is rejected for at least one n-uplet and $H_{02}$ and $H_{03}$ are not rejected for any n-uplet. Thus, a synthetic description is obtained by the distribution of these responses on a cube : at the different corners of this cube representation, are given the percentage of samples which reject one, two or three null hypotheses (Fig. 7).

The cube for $\emptyset$-samples (Fig. 7, top left) provides the expected distribution of 
the trivariate responses when no structuring patterns are simulated together with marginal confidence intervals obtained by parametric bootstrap (Efron and Tibshirani, 1993). The four other cubes in Fig. 7 clearly show other distributions in accordance with the specific characteristics of each type of simulations. Regarding the associations between the hypotheses, we clearly see on the cube of $O A$-samples that $H_{01}$ and $H_{02}$ are mainly rejected together (aggregates in occupancy and abundance are detected on the same spaces); in contrast, the situation in the case study is more complicated: there are a significant number of spaces with both types of aggregates and significant numbers of spaces with only one type (occupancy or abundance).

To summarize, the multiple test can be regarded as able to detect various types and sizes of aggregates. It works as a relevant descriptor of the structuring patterns observed on hierarchy/proximity schemes and leads finally to the decomposition of the complexity of the observed patterns.

(Figure 7 about here)

\subsection{Case study}

Aphid spatial patterns were observed on branches of Citrus trees located in Corsica (France). Each observed branch consisted of 10 successive shoots (their order being known); each shoot was formed by different numbers of leaves varying between 1 and 20. On each leaf, aphids were counted at the end of shoot growing period (beginning of June and beginning of September). As no informations are available on the orientation of each shoot, the data describing a branch are simplified into an incomplete grid (Fig. 8) satisfying the hierarchy/proximity scheme introduced above: each branch is a sample, shoots are ordered units, leaves are subunits and each subunit is weighted by the number of aphids attached.

(Figure 8 about here) 


\subsubsection{Types and scales of the aggregates}

Table 2 shows that aggregation in occupancy occurs at small and medium scales and, at less extent at large scales. In contrast aggregation in abundance only occurs at small scales. And no aggregation extending over several shoot is detected, which may result from a lack of statistical power when $H_{03}$ is tested. It has to be noticed that the null hypotheses are less often rejected than in the simulation study; this could depict that these natural branches leads to less simple structuring patterns than those simulated above.

(Table 2 about here)

\subsubsection{Associations of aggregate types}

Even if, as shown above, $H_{01}$ and $H_{02}$ are both rejected, Fig. 9 of the cube representation of the multiple test leads to a trivariate response distribution which is significantly different from the distribution for $O A$-samples. Indeed, $H_{01}$ and $H_{02}$ are often rejected alone, contrarily to what was observed for $O A$-samples. Aggregations in occupancy and in abundance are not always associated and can occur in distinct spaces. Thus, in the collection of observed branches, the complexity of aggregation figures is larger than the one produced by the simulation study: there seems to be several categories of branches in regards to aggregation (see Fig. 8 ). The future of this study would be to understood the specific features which explain this complexity.

(Figure 9 about here)

\section{Discussion \& Conclusion}

\subsection{Analysing aggregation without Euclidean distance}

In the literature, two major approaches have been explored to deal with aggregation. The first one is based on indices of variance between distant locations and aims to characterize aggregation in maps of individual abundances. Examples of tools corresponding to this approach are the variogram, the auto-covariance and the Moran's indice plotted against 


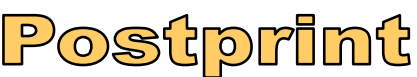

Version définitive du manuscrit publié dans / Final version of the manuscript published in : Ecological Complexity, 2009, DOI: 10.1016/j.ecocom.2009.03.012

distance (Chilès and Delfiner 1999; Cressie, 1991). Note that indices of variance such as the $F_{S T}$ statistic also exist for analyzing spatial structuring in genetic maps (Epperson, 2003), but in this communication our interest is restricted to demographic data, that is to say occupancy and abundance data.

The second approach is based on deviance from complete spatial randomness (CSR), and aims to characterize aggregation in patterns of points, the points being the locations of (groups of) individuals. Most of the corresponding tools use the intensity function of points and functions of the inter-event distances as the $K$-function (Diggle, 2003, Illian et al., 2008). For example, a variation in the intensity or an excess of short inter-point distances will be interpreted as signs of aggregation. Note that it is possible to look at deviances from spatial randomness in the context of an inhomogeneous intensity (Brix et al., 2001); in this case the aim is to study a sort of double clustering structure.

In this article, we have presented a multiple test of aggregation which aims to overcome the absence of classical distance measurements required by the standard methods described above. Instead of distance we have described the space by two "dimensions" : proximity and hierarchy so to capture explicit spatial structuring. These two components allow us to give a framework for understanding the "similarity" between different locations in a given space and the multiple test carries the possibility to decompose the similarity following its strictly spatial component (also called "occupancy" in the paper) and its abundance component. It is usual to address structuring pattern by the discrepancy of one observed sample from the Complete Spatial Randomness (CSR) hypothesis (Diggle, 2003). The reference to CSR limits the approach of structuring patterns because it is an extreme case of "no pattern" and because it is adapted to global structuring patterns. When the CSR is taken as null hypothesis to test aggregation in the simulated sample presented here, almost $100 \%$ of the sample are rejecting the null hypothesis. Additionally, it is relevant to consider that all locations of the space are not available to the presence of the populations under study. A Partial Spatial Randomness (PSR) has been approached by using permutation tests. The characterisation of structuring relies then of the resistance to permutation, i.e. the fact that the observed sample is located 
outside the cluster of the permuted samples in a given space of parameters.

Finally, each dimension of the multiple test realizes either the similarity component responsible of the structuring (occupancy or abundance) or the hierarchical scale at which takes place the structuring. The finer the hierarchical scale, the more precise the scale at which aggregation is detected. The hierarchy has been defined here at the uplet scale and by the hierarchical level from sub-units to units.

The simulation study has shown the applicability of the multiple test and has allowed us to clarify the properties and the limits of this approach.

\subsection{Decomposing the complexity of aggregation}

The multiple test in the framework of PSR reference leads to a pertinent decomposition of the complexity of structuring modes. As each of the test step provide a vision of structuring, the multiple test procedure enlightens a multiple structuring which leads to a better understanding of how the final spatial structure is built, with a dominance of occupancy characteristics or of the abundance properties and at which uplet scale (or hierarchical level) the structuring pattern is relevant to be considered. If the complexity of patterns does not allow to identify individually the patterns or the aggregate itself, the multiple test provides an evaluation of the dimension at which aggregates could be detected. In absence of distance criteria and with no constraint on the model for marginal distribution of the individual characteristics, the multiple procedure is a guide to focus on the dimension at which the structuring pattern should be modelled. Additionally, in giving the different hypotheses which are rejected by a given spatialized population sample, it attests to the constraints to be followed in the modelling approaches to be developed.

We have already noticed in the introduction that aggregation can be analysed in hierarchical spaces involving a large number of levels while only two have been considered in the case study presented above. In the former case questions about self-similarity arise: Is aggregation present at every level? Is it the same type of aggregation which is observed? Fractal methods are currently used to bring responses to such questions 
1 (Ferrandino 2004; Mandelbrot 1977; Storch et al 2008) but in any case they require the definition of a distance. Without distance but with the notion of proximity as defined 3 in our approach, we could investigate the questions about self-similarity.

It is to be noticed that, in this study, the units are ordered on a one-dimensional axis. This limitation, which works in the case study, could be overcome by a more complex definition of the relationships between units. The procedure in itself would not be modified or invalidated by a more complex definition of spatial proximity as the neibourhood systems proposed in Rue and Held (2005); it could be even considered that 9 it is a property of this approach to allow to account for spatial complexity by the way of the definition of hierarchy/proximity structure.

\subsection{Ecological perspectives}

The main results in the case study on aphids may be linked to ecological processes given rise to the observed patterns. For example, the existence at a significant level of the four types of patterns $(\emptyset, O, A$ and $O A)$ could illustrate the diversity in the results of the interaction between the aphid dynamics and the irregularity of the spatial structure of the host plant. The small aggregates in abundance ( $A$ aggregates) could be linked with the reproduction component of the aphid dynamics which occurs at a very local scale (fundation effect due to the few reproductive females). The aggregates in occupancy $(O$ aggregates), which are larger than the $A$-ones, could be linked with the dispersal ability of offsprings.

These qualitative links which are made between our results and the ecological processes could be strengthened:

- by spatiotemporal surveys (the reproductions, the migrations, the formation of aggregates would be followed with time) and/or

- by a model selection approach (selecting a model of the population dynamics which gives rise to the same type of patterns than the observed ones).

Regarding the second point, we mentioned in the introduction the difficulty of construct- 
1 ing a model for aphid structuring patterns because of their complexity. The present study should be valuable in this perspective in providing assumptions for the constraints to be incorporated in the model fitting procedure (Grimm et al., 2005; , Wiegand et al. 2003) 4 using minimum contrast estimation (Dacunha-Castelle and Duflo, 1982 ; Soubeyrand et al., 2008a) or approximate Bayesian computation (Beaumont et al., 2002).

A last remark to emphasize that the method explored in this paper and the results of such approach do not depend by any way on the type of biological components (defining the space as well as the individuals). It is indeed possible to generalize this procedure, the quality of which relies essentially on (i) the pertinent choice of null hypotheses of the multiple test in order to capture specificities of the strategies which individuals spread and on (ii) the suitability of the hierarchical description to account for the specificity of the space under study, both being the premisses of more accurate models based on times series. It is to be noticed that the hierarchy considered in this article (leaf, branch) can be extended in the range of levels considered (cell, leaf, branch, tree, orchard; see Soubeyrand et al 2007c) and even exported to other contexts, e.g. administritive hierarchical structures in human epidemiological surveys (Henderson et al 2002) and insect dynamics surveys (Soubeyrand et al 2009).

\section{Acknowledgments}

The data which were used here in the case study have been collected by INRA, Unité ROSE, 400 route des Chappes, 06903 Sophia Antipolis, France. We warmly thank L. Lapchin and R. Boll for introducing us to these data and for the fruitful discussions about aggregation in aphids ecology. We are also very grateful to the colleagues at Biostatistics and Spatial Processes laboratory for their availability and comments about this work. 


\section{Appendix}

$2 O A-, O-, A$ - and $\emptyset$-samples were simulated by combining three layers defined in this 3 appendix. R programs (lines starting with $>$ ) show how the layers were obtained and 4 combined. 75 to 25 .

8

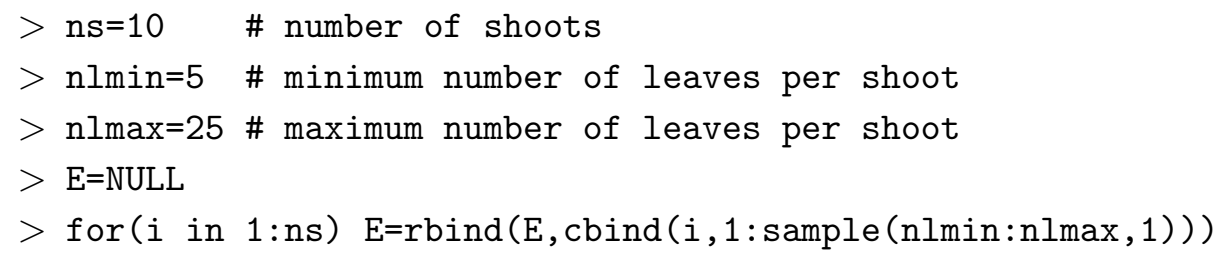

Layer two: the occupancy structure $O: \mathbb{E} \rightarrow\{0,1\}$ was defined as the excursion set of a Gaussian random process (Lantuéjoul, 2002): that is to say for all $e \in \mathbb{E}, O(e)$ equals one if $Z(e)>0$ and zero else, where $Z: \mathbb{E} \rightarrow \mathbb{R}$ denotes the Gaussian process defined over $\mathbb{E}$ and with values in $\mathbb{R}$. To obtain aggregated "ones" and aggregated "zeros", a Gaussian auto-correlation function was used to simulate the Gaussian process (Chilès and Delfiner, 1999).

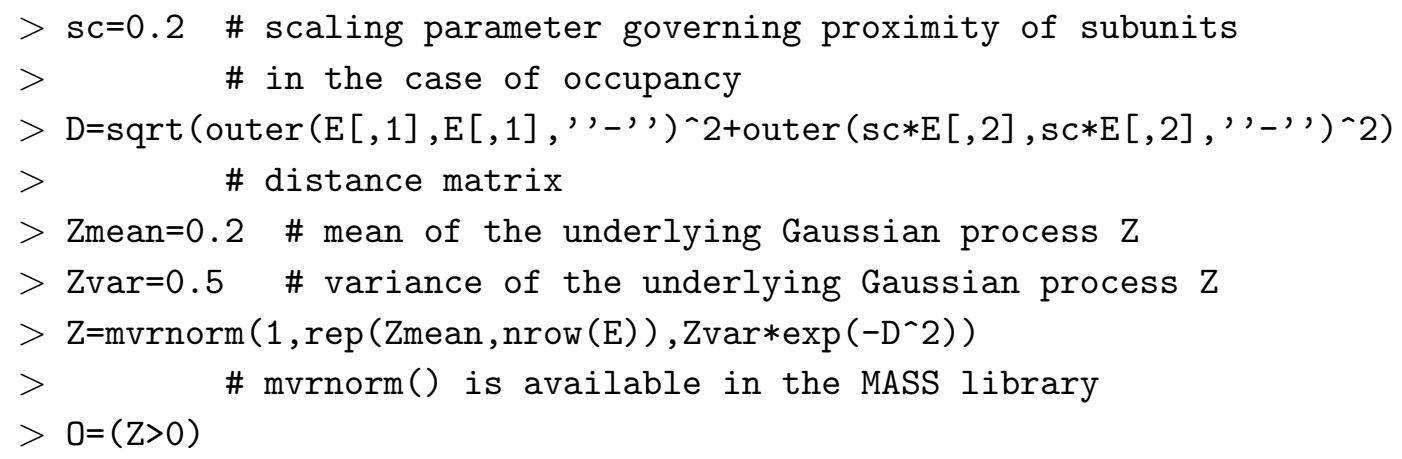

Layer three: the abundance structure $A: \mathbb{E} \rightarrow \mathbb{N}$ was based on a doubly stochastic random process (Diggle, 2003): A log-Gaussian random process $Y: \mathbb{E} \rightarrow \mathbb{R}_{+}$with a Gaussian auto-correlation function was first simulated; Then, the abundance $A(e)$ in 
1 subunit $e \in \mathbb{E}$ was drawn from a Poisson distribution with mean value $Y(e)$ to which 2 the value one is added (to avoid null abundances). The spatial structuring of $Y$ allowed us to get a spatially structured abundance process $A$. Remark: This model can also be written in the framework of spatial generalized linear mixed models (Diggle et al. 1998).

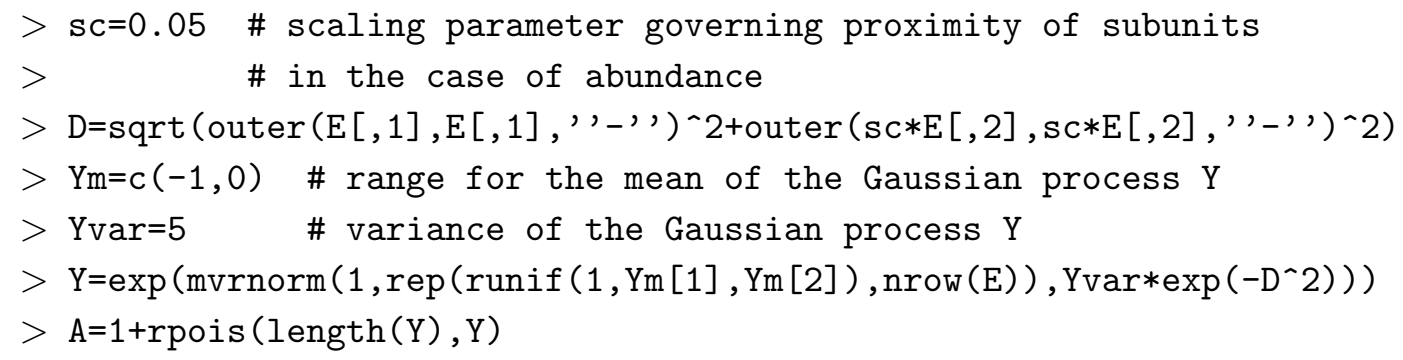

Then, the three layers are combined as follows to get the four sorts of samples presented in the simulation study.

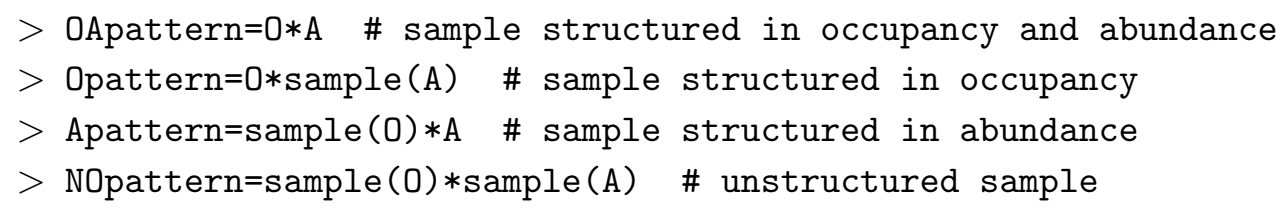

\section{References}

Baddeley, A., 2008. Analysing spatial point patterns in R. Workshop notes. CSIRO eds. http://www.csiro.au/resources/pf16h.html

Bar-Hen, A. and Picard, N., 2007. Simulation study of dissimilarity between point process. Computational Statistics, 21; 487-507.

Beaumont, M. A., Zhang, W. and Balding, D. J., 2002. Approximate Bayesian Computation in Population Genetics. Genetics, 162; 2025-2035.

Brix, A., Senoussi, R., Couteron, P. and Chadœuf, J., 2001. Assessing Goodness of Fit of Spatially Inhomogeneous Poisson Processes. Biometrika, 88; 487-497.

Chambers, J. M., Freeny, A. and Heiberger, R. M., 1992. Analysis of variance; designed experiments. In: J. M. Chambers and T. J. Hastie (editors), Statistical Models in S. 
1 Wadsworth and Brooks/Cole, Pacific Grove, pp.145-194

2 Chilès, J.-P. and Delfiner, P., 1999. Geostatistics. Modeling Spatial Uncertainty. Wiley, 3 New York, 695 pp.

4 Conlisk, E., Bloxham, M., Conlisk, J., Enquist, B. and Harte, J., 2007. A new class of 5 models of spatial distribution. Ecological Monography, 77 (2); 269:284.

6 Cressie, N. A. C., 1991. Statistics for Spatial Data. Wiley, New York, 900 pp.

Dacunha-Castelle, D. and Duflo M., 1982. Probabilités et statistiques, tome 2, Problèmes à temps mobile. Masson, Paris, 286 pp.

Diggle, P. J., 2003. Statistical Analysis of Spatial Point Patterns (2nd ed.), Academic - Press, New York, 159 pp.

11 Diggle, P. J., Tawn, J. A. and Moyeed, R. A., 1998. Model-based geostatistics. Applied 12 Statistics, 47; 299-350.

Efron, B. and Tibshirani, R. J., 1993. An introduction to the bootstrap. Chapman \& Hall. New York, 436 pp.

Epperson, B. K., 2003. Geographical genetics. Princeton University Press, 369 pp.

Ferrandino, F.J., 2004. Analytical and Theoretical Plant Pathology Measuring Spatial Aggregation in Binary Epidemics: Correlative Analysis and the Advantage of FractalBased Sampling. Phytopathology 94: 1215-1227.

Gilbert, M., Fielding, N., Evans, H.F. and Gregoire, J.-C., 2003. Spatial pattern of invading Dendroctonus micans (Coleoptera : Scolytidae) populations in the United Kingdom. Can. J. For. Res., 33(4); 712:725

Grimm, V., Revilla, E., Berger, U., Jeltsch, F., Mooij, W. M., Railsback, S. F., Thulke, H-H.,Weiner, J., Wiegand, T. and DeAngelis, D. L., 2005. Pattern-oriented modeling of agent-based complex systems : lesson for ecology. Science, 310; 987-991

Henderson, R., Shimakura, S. and Gorst, D.; 2002. Modeling spatial variation in leukemia survival data. Journal of the American Statistical Association, 97; 965-972.

Illian, J., Penttinen, A., Stoyan, H. and Stoyan, D., 2008. Statistical Analysis and Modelling of Spatial Point Patterns. Wiley, New York.

Inouye, B. D., 2005. Scaling up from local competition to regional coexistence across 
1 two scales of spatial heterogeneity : insect larvae in the fruits of Apeiba membranacea . Oecologia, 145; 188-196.

Johst, K., Brandl, R., Eber, S., 2002. Metapopulations persistence in dynamic landscapes : the role of dispersal distance. Oikos, 98; 263-270.

5 Keitt, T.H., Bjørnstad,O., Dixon, P.M. and Citron-Pousty, S., 2002. Accounting for spatial pattern when modeling organism-environment interactions. Ecography, 25; 616-625.

Laine, A.-L. and Hanski, I., 2006. Large-scale spatial dynamics of specialist plant pathogen. Journal of Ecology, 94; 217-226.

Lantuéjoul, C., 2002. Geostatistical Simulation, Models and Algorithms. Springer, o Berlin, $256 \mathrm{pp}$.

11 Levin, S., 1992. The problem of pattern and scale in ecology. Ecology, 73(6); 1943-1967 12 Mandelbrot, B.B., 1977. The Fractal Geometry of Nature. Freeman, San Francisco.

Manly, B.F.J., 1997. Randomization, bootstrap and Monte Carlo methods in Biology. (2nd ed.), Chapman and Hall, London, 281 pp.

Oddou-Muratorio, S., Demesure-Musch, B., Pelissier, R. and Gouyon, P.-H., 2004. Impacts of gene flows and logging history on the local genetic structure of a scattered tree species Sorbus torminalis L. Crantz. Molecular Ecology, 13; 3689-3702.

Perry, J.N., Liebhold, A.M., Rosenberg, M.S., Miriti, J., Jakomulska, A. and CitronPousty, S., 2002. Illustrations and guidelines for selecting statistical methods for quantifying spatial pattern in ecological data. Ecography, 25; 578-600.

Rue, H. and Held, L., 2005. Gaussian markov random fields. Theory and applications. Chapmann and Hall, New York, 263 pp.

Soubeyrand, S., Enjalbert, J., Sanchez, A. and Sache, I., 2007a. Anisotropy, in density and in distance, of the dispersal of yellow rust of wheat: Experiments in large field plots and estimation. Phytopathology, 97; 1315-1324.

Soubeyrand, S, Sache, I., Lannou, C. and Chadœuf, J., 2007b. A frailty model to assess plant disease spread from individual count data. Journal of Data Science, 5; 67-83.

Soubeyrand, S., Thébaud, G. and Chadœuf, J., 2007c. Accounting for biological variability and sampling scale: a multi-scale approach to building epidemic models. Journal 
1 of the Royal Society Interface 4: 985-997.

2 Soubeyrand, S., Carpentier, F., Desassis, N. and Chadœuf, J., 2008a. Incorporating a 3 contrast in the Bayesian formula: What consequences for the MAP estimator and the 4 posterior distribution? Applications in spatial statistics. arXiv:0804.4780v1

5 Soubeyrand, S., Held, L. Höhle, M. and Sache, I., 2008b. Modelling the spread in space 6 and time of an airborne plant disease. Applied Statistics, 57; 253-272.

7 Soubeyrand, S., Neuvonen, S. and Penttinen, A., 2009. Mechanical-statistical modelling in ecology: from outbreak detections to pest dynamics. Bulletin of Mathematical Biology 71: $318-338$.

10 Storch, D., Scaronizling, A.L., Reif, J., Polechová, J., Scaronizlingová, E. and Gaston, 11 K.J., 2008. The quest for a null model for macroecological patterns: geometry of species 12 distributions at multiple spatial scales. Ecological Letters 11: 771-784.

13 Tilman, D. and Kareiva, P., 1996. Spatial Ecology: The Role of Space in Population

Dynamics and Interspecific Interactions. Princeton Univ. Press, Princeton, 368 pp.

Virtanen, T., Neuvonen, S., Nikula, A., Varama, M. and Niemelä, P., 1996. Climate change and the risks of Neodiprion sertifer outbreaks on Scots pine. Silva Fennica, 30; 169-177.

Wiegand, T., Jeltsch, F., Hanski, I. and Grimm, V., 2003. Using pattern-oriented modeling for revealing hidden information: a key for reconciling ecological theory and application. Oikos, 100; 209-222. 


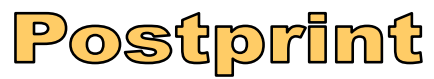

Version définitive du manuscrit publié dans / Final version of the manuscript published in : Ecological Complexity, 2009, DOI: 10.1016/j.ecocom.2009.03.012

Table 1

\begin{tabular}{c|c|ccccccccc}
\hline \multirow{2}{*}{ Types } & Hypotheses & \multicolumn{1}{|c}{ n-uplets } & & & \\
& & $\mathrm{n}=1$ & $\mathrm{n}=2$ & $\mathrm{n}=3$ & $\mathrm{n}=4$ & $\mathrm{n}=5$ & $\mathrm{n}=6$ & $\mathrm{n}=7$ & $\mathrm{n}=8$ & $\mathrm{n}=9$ \\
\hline \multirow{3}{*}{$O A$} & Freq $H_{01} \leq 0.05$ & $63^{\star}$ & $69^{\star}$ & $69^{\star}$ & $60^{\star}$ & $51^{\star}$ & $51^{\star}$ & $48^{\star}$ & $42^{\star}$ & $43^{\star}$ \\
& Freq $H_{02} \leq 0.05$ & $70^{\star}$ & $68^{\star}$ & $57^{\star}$ & $56^{\star}$ & $46^{\star}$ & $36^{\star}$ & $32^{\star}$ & $18^{\star}$ & 7 \\
& Freq $H_{03} \leq 0.05$ & 0 & 1 & 3 & 5 & 4 & 5 & 6 & 2 & 1 \\
\hline \multirow{5}{*}{$O$} & Freq $H_{01} \leq 0.05$ & $63^{\star}$ & $68^{\star}$ & $69^{\star}$ & $60^{\star}$ & $52^{\star}$ & $52^{\star}$ & $49^{\star}$ & $42^{\star}$ & $42^{\star}$ \\
& Freq $H_{02} \leq 0.05$ & 4 & 5 & 4 & 8 & 7 & 8 & 8 & 5 & 5 \\
& Freq $H_{03} \leq 0.05$ & 0 & 4 & 3 & 1 & 4 & 5 & 5 & 4 & 2 \\
\hline \multirow{5}{*}{$A$} & Freq $H_{01} \leq 0.05$ & 9 & 5 & 4 & 3 & 7 & 4 & 6 & 3 & 5 \\
& Freq $H_{02} \leq 0.05$ & $56^{\star}$ & $60^{\star}$ & $57^{\star}$ & $49^{\star}$ & $49^{\star}$ & $31^{\star}$ & $30^{\star}$ & $13^{\star}$ & 7 \\
& Freq $H_{03} \leq 0.05$ & 0 & 1 & 2 & 5 & 4 & 3 & 2 & 4 & 3 \\
\hline \multirow{3}{*}{$\emptyset$} & Freq $H_{01} \leq 0.05$ & 3 & 4 & 5 & 3 & 3 & 1 & 6 & 6 & 4 \\
& Freq $H_{02} \leq 0.05$ & 4 & 7 & 4 & 3 & 5 & 7 & 5 & 4 & 3 \\
& Freq $H_{03} \leq 0.05$ & 0 & 3 & 1 & 2 & 3 & 3 & 6 & 4 & 1 \\
\hline \multirow{3}{*}{$L A R G E$} & Freq $H_{01} \leq 0.05$ & $33^{\star}$ & $53^{\star}$ & $65^{\star}$ & $69^{\star}$ & $64^{\star}$ & $59^{\star}$ & $42^{\star}$ & $31^{\star}$ & $24^{\star}$ \\
& Freq $H_{02} \leq 0.05$ & $96^{\star}$ & $95^{\star}$ & $91^{\star}$ & $93^{\star}$ & $94^{\star}$ & $86^{\star}$ & $82^{\star}$ & $69^{\star}$ & $31^{\star}$ \\
& Freq $H_{03} \leq 0.05$ & 0 & 1 & 3 & $15^{\star}$ & $23^{\star}$ & 2 & 0 & 1 & 0 \\
\hline
\end{tabular}

Table 1: Number of $O A-, O-, A$ - and $\emptyset$-samples and of $L A R G E$ aggregate samples, the p-value of which are $\leq 0.05$ at increasing size of uplet, for the three different null hypotheses. ${ }^{\star}$ : significancy at the level of $5 \%$ by comparison with a draw in $B(100,0.05)$. 


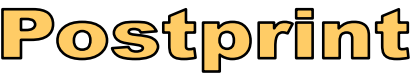

Version définitive du manuscrit publié dans / Final version of the manuscript published in : Ecological Complexity, 2009, DOI: 10.1016/j.ecocom.2009.03.012

1

Table 2

\begin{tabular}{c|c|ccccccccc}
\hline Type & n-uplet & $\mathrm{n}=1$ & $\mathrm{n}=2$ & $\mathrm{n}=3$ & $\mathrm{n}=4$ & $\mathrm{n}=5$ & $\mathrm{n}=6$ & $\mathrm{n}=7$ & $\mathrm{n}=8$ & $\mathrm{n}=9$ \\
\hline \multirow{5}{*}{ Branches } & Freq $H_{01} \leq 0.05$ & $33^{\star}$ & $31^{\star}$ & $30^{\star}$ & $28^{\star}$ & $34^{\star}$ & $23^{\star}$ & $16^{\star}$ & $14^{\star}$ & $10^{\star}$ \\
& Freq $H_{02} \leq 0.05$ & $24^{\star}$ & $20^{\star}$ & $15^{\star}$ & $12^{\star}$ & $11^{\star}$ & 6 & 5 & 2 & 0 \\
& Freq $H_{03} \leq 0.05$ & 0 & 2 & 3 & 5 & 3 & 2 & 3 & 1 & 4 \\
\hline
\end{tabular}

Table 2: Percentage of branches the p-value of which are $\leq 0.05$ at increasing size of uplet, for the three different null hypotheses. * : significancy at the level of $5 \%$ by comparison with a draw in $B(160,0.05)$. 


\section{List of Figures}

1 Hierarchical spaces made of subunits (dots) nested in units (thin lines on the left and rectangles on the right). Proximities between units are indicated by the thick segments. Remark: degrees of proximity between subunits can be defined on the left (this scheme may represent the system leaves-shoots-branch) but not necessarily on the right (this scheme may represent the system branch-tree-orchard) . . . . . . . . . . . . . . . 26

2 Typical ways of aggregation in occupancy (filled squares on the 2D grid) and/or abundance (red tall bars for hight values; yellow small bars for low values). a: no aggregation; b: aggregation in abundance; c: aggregation in occupancy; d: aggregation in abundance and occupancy.

3 Examples of permutations applied to an occupancy pattern at the subunit level (top), to an abundance pattern at the subunit level (centre) and to an abundance pattern at the unit level (bottom). . . . . . . . . . 28

4 Decomposition of an hypothetical space with four units into the three 2-uplets and computation of the test statistics $S_{n}, n=2 \ldots \ldots 29$

5 Observed statistic $S_{n}=\left(\mu_{n k^{\star}}, \sigma_{n k^{\star}}^{2}\right)$ for a given pattern of a given space (large dot) and statistics computed for 5,000 permuted patterns (small dots). The rectangle centred around the observed statistic defines its surrounding used in the computation of the test p-value. . . . . . . . . . 30

6 Schematic images of $\emptyset$-, $O A$-, $O-, A$ - and $L A R G E$-samples (red circle : occupied subunits, sizes proportional to the abundances indicated with black numbers; green diamond : unoccupied subunits) . . . . . . . . . . . 31 


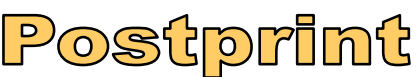

Version définitive du manuscrit publié dans / Final version of the manuscript published in : Ecological Complexity, 2009, DOI: 10.1016/j.ecocom.2009.03.012

7 Cube decomposition of the multiple test results for $\emptyset_{-}, O A_{-}, O_{-}, A_{-}$and $L A R G E$-samples. In the multiple test procedure, three hypotheses with two modalities ( $0=$ accepted: $H_{0 i}$ and $1=$ rejected: $\left.\bar{H}_{0 i}\right)$ are tested and eight different trivariate responses are expected $((0,0,0),(1,0,0),(0,1,0)$, $(0,0,1),(1,1,0),(1,0,1),(0,1,1)$ and $(1,1,1))$. On the cube representation each direction carries one hypothesis and each corner carries one trivariate response. The number at each corner gives the percentage of samples for which the respective trivariate response is obtained.

8 Schematic images of four real branches (the construction of the schematic images is explained in Fig. ??). The four branches have been chosen because they respond differently to the multiple test and correspond to the four types defined in the simulation study : $\emptyset$-branch $=$ no one of the three hypotheses rejected; $O A$-branch $=H_{01}$ and $H_{02}$ are both rejected; $O$-branch $=H_{01}$ only is rejected; $A$-branch $=H_{02}$ only is rejected. $\ldots . .33$

9 Cube decomposition of the multiple test results for the real branches. The construction of the cube is explained in Fig. ?? . . . . . . . . . . . . . . 34 


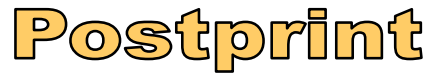

Version définitive du manuscrit publié dans / Final version of the manuscript published in : Ecological Complexity, 2009, DOI: 10.1016/j.ecocom.2009.03.012
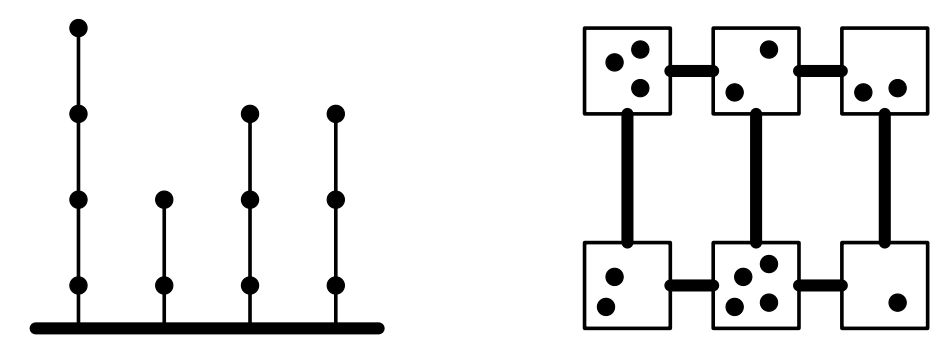

Figure 1: Hierarchical spaces made of subunits (dots) nested in units (thin lines on the left and rectangles on the right). Proximities between units are indicated by the thick segments. Remark: degrees of proximity between subunits can be defined on the left (this scheme may represent the system leaves-shoots-branch) but not necessarily on the right (this scheme may represent the system branch-tree-orchard). 


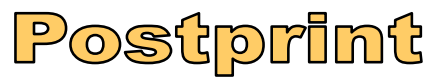

Version définitive du manuscrit publié dans / Final version of the manuscript published in : Ecological Complexity, 2009, DOI: 10.1016/j.ecocom.2009.03.012
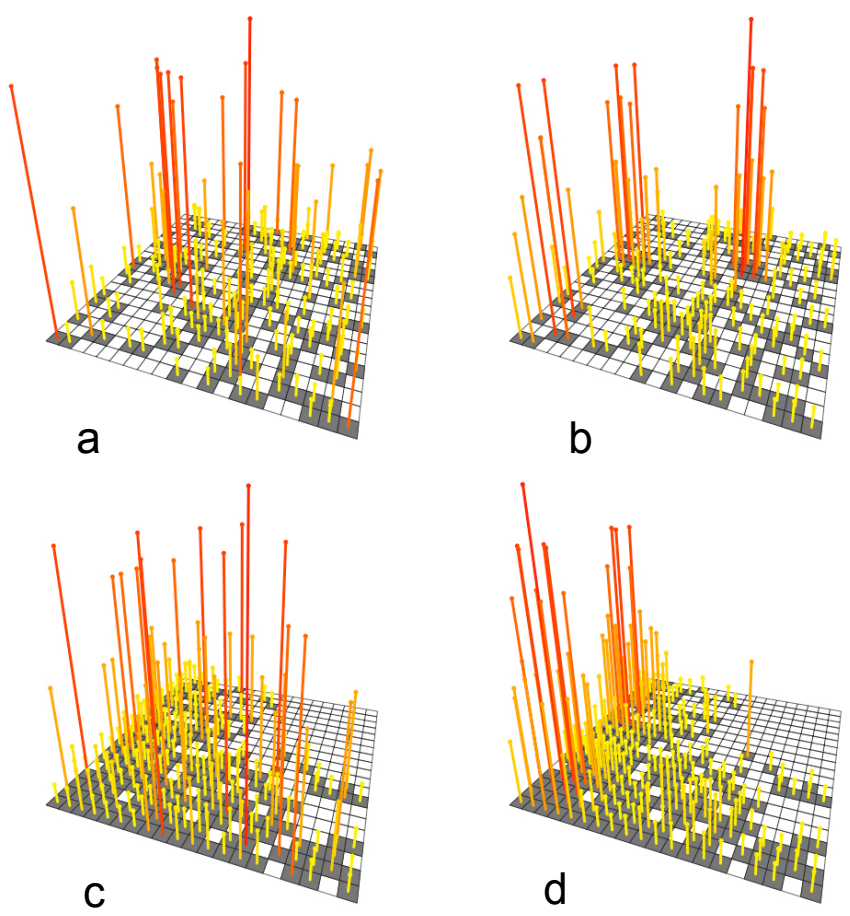

Figure 2: Typical ways of aggregation in occupancy (filled squares on the 2D grid) and/or abundance (red tall bars for hight values; yellow small bars for low values). a: no aggregation; b: aggregation in abundance; c: aggregation in occupancy; d: aggregation in abundance and occupancy. 


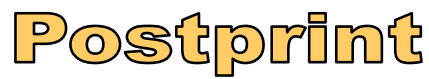

Version définitive du manuscrit publié dans / Final version of the manuscript published in : Ecological Complexity, 2009, DOI: 10.1016/j.ecocom.2009.03.012
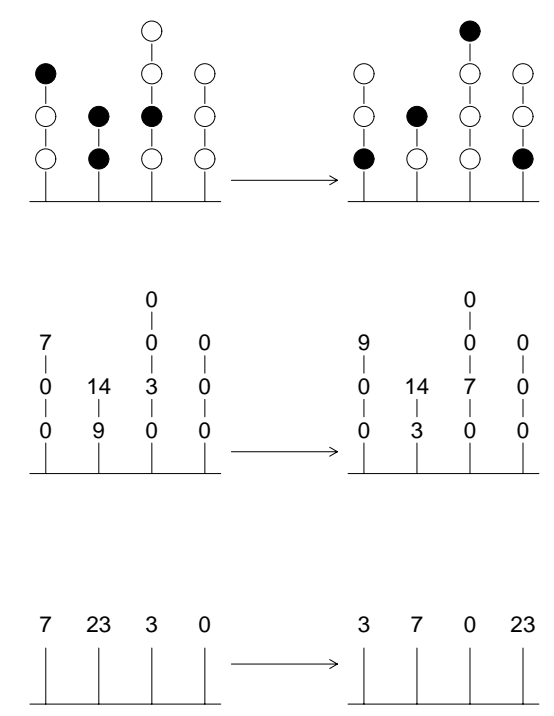

Figure 3: Examples of permutations applied to an occupancy pattern at the subunit level (top), to an abundance pattern at the subunit level (centre) and to an abundance pattern at the unit level (bottom). 


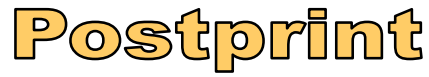

Version définitive du manuscrit publié dans / Final version of the manuscript published in : Ecological Complexity, 2009, DOI: 10.1016/j.ecocom.2009.03.012

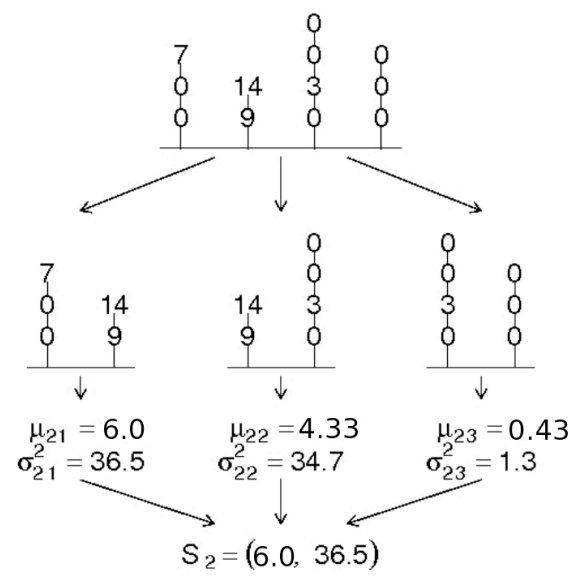

Figure 4: Decomposition of an hypothetical space with four units into the three 2-uplets and computation of the test statistics $S_{n}, n=2$. 


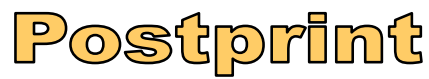

Version définitive du manuscrit publié dans / Final version of the manuscript published in : Ecological Complexity, 2009, DOI: 10.1016/j.ecocom.2009.03.012

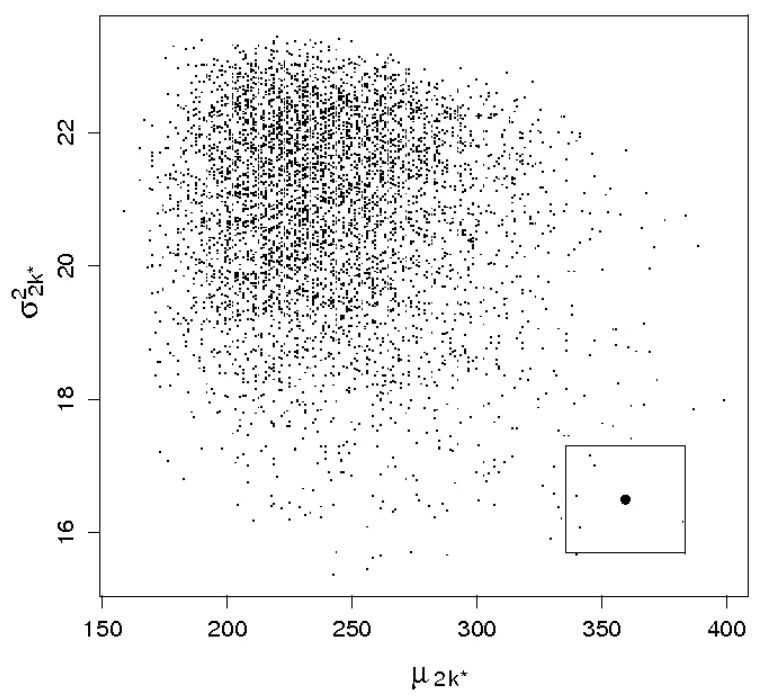

Figure 5: Observed statistic $S_{n}=\left(\mu_{n k^{\star}}, \sigma_{n k^{\star}}^{2}\right)$ for a given pattern of a given space (large dot) and statistics computed for 5,000 permuted patterns (small dots). The rectangle centred around the observed statistic defines its surrounding used in the computation of the test p-value. 


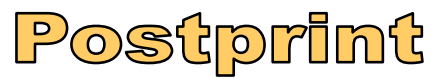

Version définitive du manuscrit publié dans / Final version of the manuscript published in : Ecological Complexity, 2009, DOI: 10.1016/j.ecocom.2009.03.012
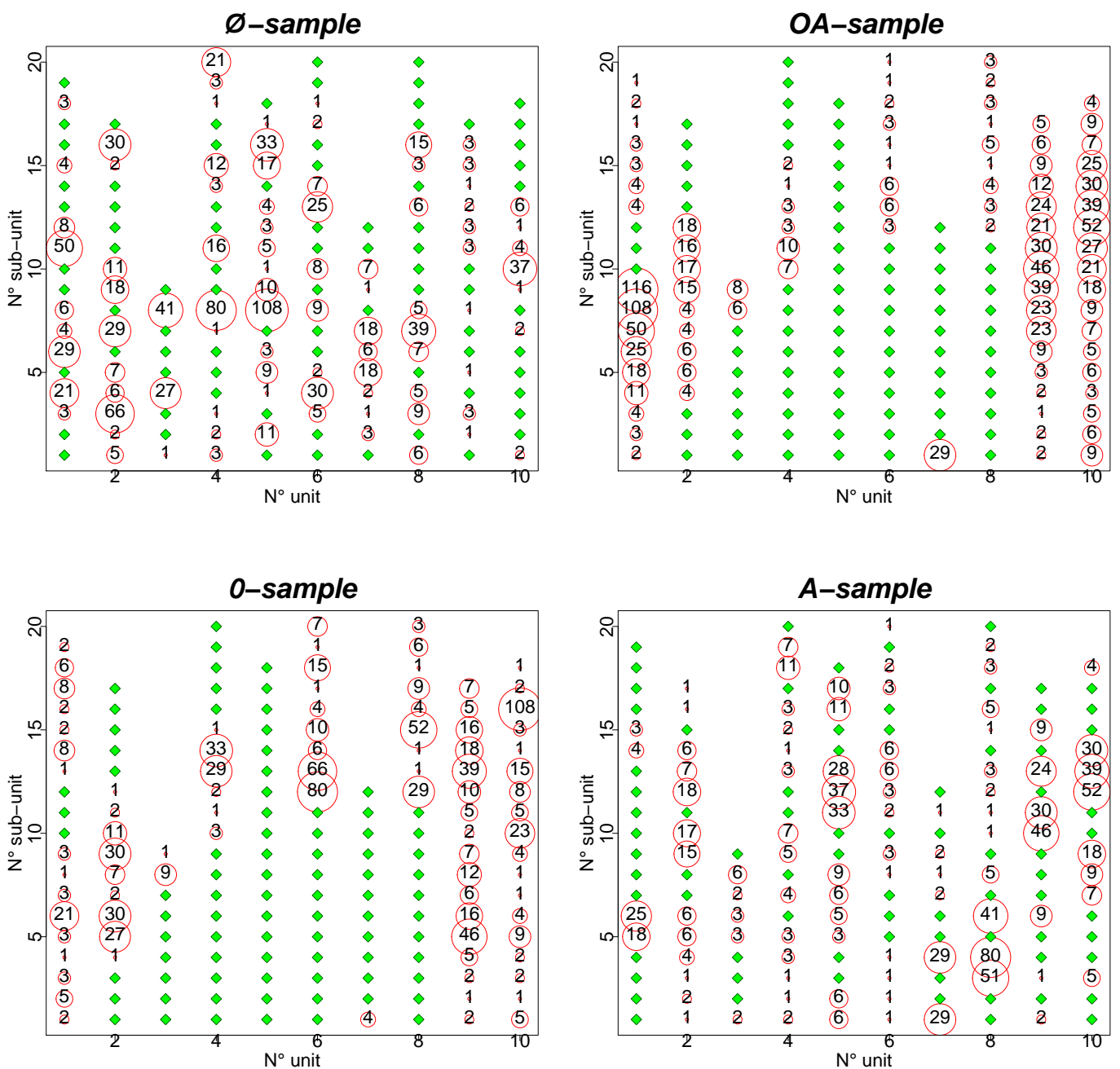

\section{LARGE}

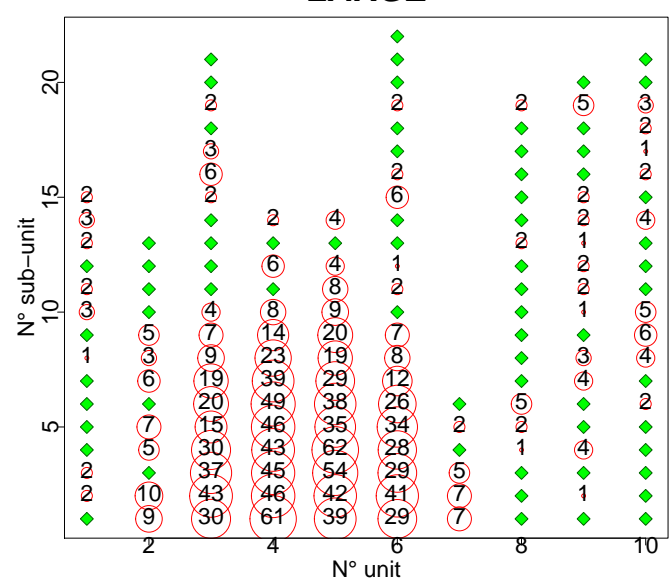

Figure 6: Schematic images of $\emptyset_{-}, O A-, O-, A$ - and $L A R G E$-samples (red circle : occupied subunits, sizes proportional to the abundances indicated with black numbers; green diamond : unoccupied subunits). 


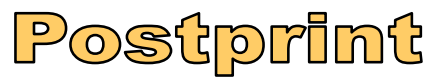

Version définitive du manuscrit publié dans / Final version of the manuscript published in : Ecological Complexity, 2009, DOI: 10.1016/j.ecocom.2009.03.012
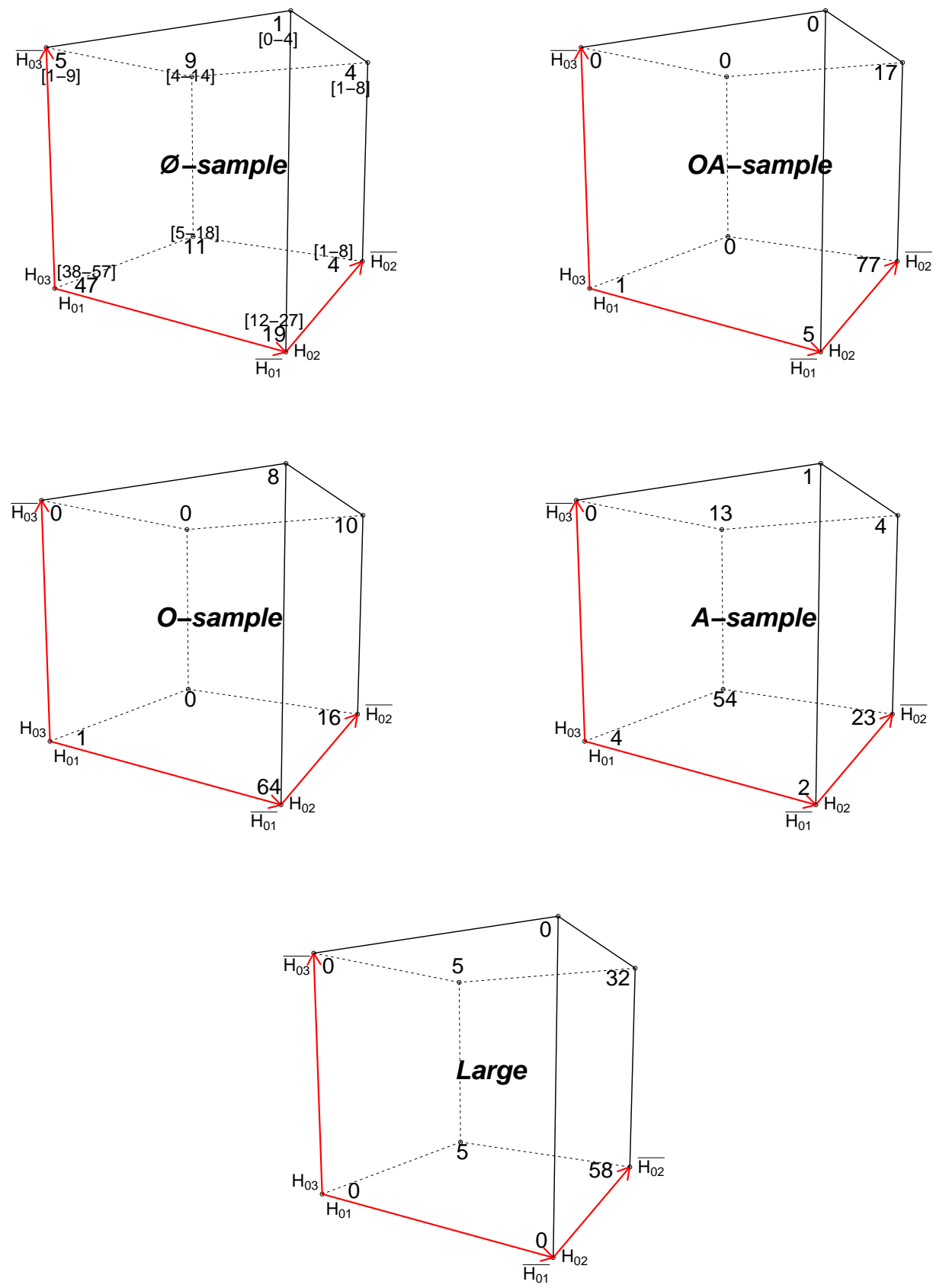

Figure 7: Cube decomposition of the multiple test results for $\emptyset_{-}, O A_{-}, O_{-}, A$ - and $L A R G E$-samples. In the multiple test procedure, three hypotheses with two modalities ( $0=$ accepted: $H_{0 i}$ and $1=$ rejected: $\left.\bar{H}_{0 i}\right)$ are tested and eight different trivariate responses are expected $((0,0,0),(1,0,0),(0,1,0),(0,0,1),(1,1,0),(1,0,1),(0,1,1)$ and $(1,1,1))$. On the cube representation each direction carries one hypothesis and each corner carries one trivariate response. The number at each corner gives the percentage of samples for which the respective trivariate response is obtained. 


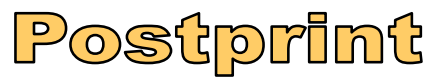

Version définitive du manuscrit publié dans / Final version of the manuscript published in : Ecological Complexity, 2009, DOI: 10.1016/j.ecocom.2009.03.012
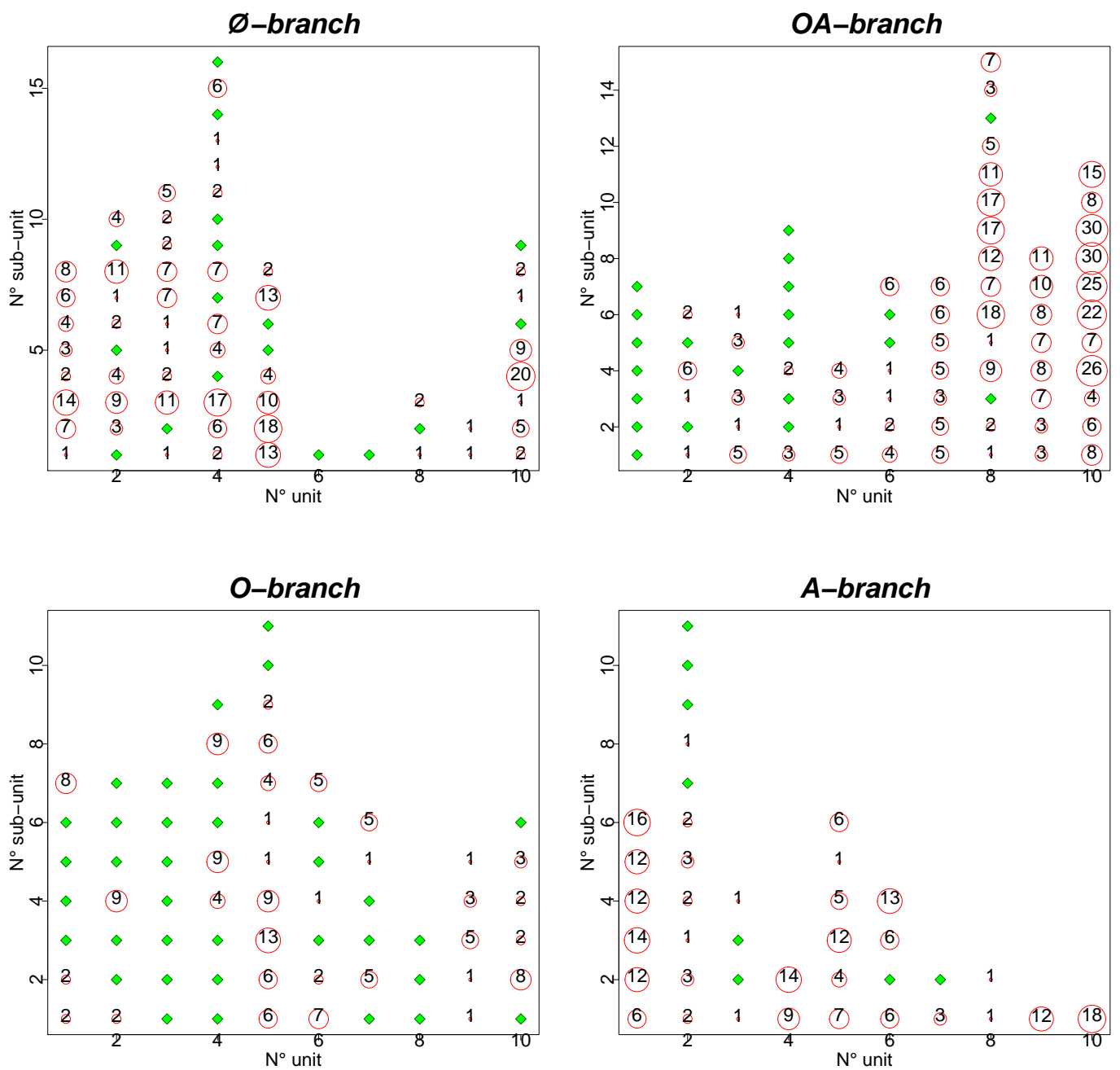

Figure 8: Schematic images of four real branches (the construction of the schematic images is explained in Fig. 6). The four branches have been chosen because they respond differently to the multiple test and correspond to the four types defined in the simulation study : $\emptyset$-branch $=$ no one of the three hypotheses rejected; $O A$-branch $=H_{01}$ and $H_{02}$ are both rejected; $O$-branch $=H_{01}$ only is rejected; $A$-branch $=H_{02}$ only is rejected. 


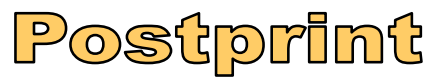

Version définitive du manuscrit publié dans / Final version of the manuscript published in : Ecological Complexity, 2009, DOI: 10.1016/j.ecocom.2009.03.012

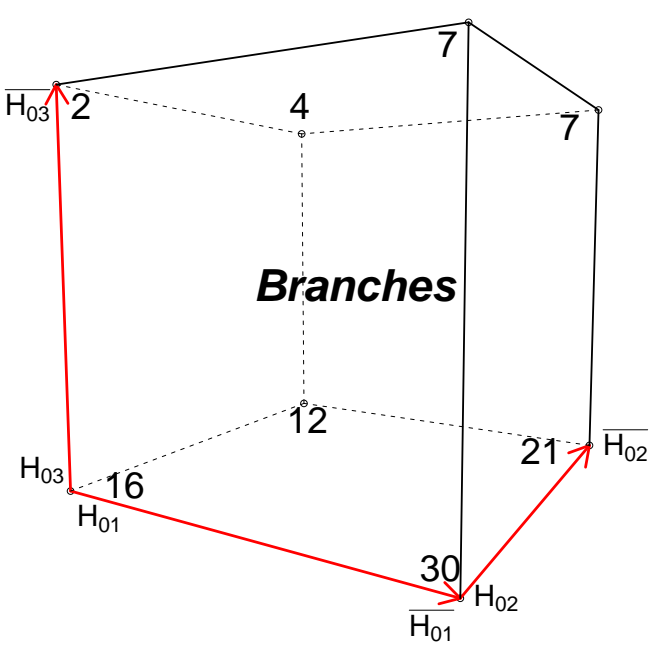

Figure 9: Cube decomposition of the multiple test results for the real branches. The construction of the cube is explained in Fig. 7. 